\title{
DINÂMICA DE CRESCIMENTO E PRODUTIVIDADE DE FORRAGEM DE Trachypogon plumosus SOB NÍVEIS DE CORREÇÃO DA FERTILIDADE DO SOLO E IDADES DE REBROTA
}

\section{GROWTH DYNAMIC AND FORAGE YIELD OF Trachypogon plumosus UNDER LEVELS OF SOIL FERTILITY CORRECTION AND REGROWTH AGES}

\author{
Newton de Lucena Costa ${ }^{1^{*}}$ \\ Anibal de Moraes $^{2}$ \\ Paulo César de Faccio Carvalho ${ }^{3}$ \\ Alda Lúcia Gomes Monteiro ${ }^{4}$ \\ Antônio Carlos Vargas Motta ${ }^{5}$ \\ Ricardo Augusto de Oliveira²
}

\begin{abstract}
${ }^{1}$ Embrapa Roraima, Boa Vista, RR, Brasil.
${ }^{2}$ Departamento de Fitotecnia e Fitossanitarismo, Universidade Federal do Paraná, Curitiba, PR, Brasil. 3Departamento de Plantas Forrageiras e Agrometeorologia Universidade Federal do Rio Grande do Sul, Porto Alegre, RS, Brasil.

${ }^{4}$ Departamento de Zootecnia, Universidade Federal do Paraná, Curitiba, PR, Brasil.

${ }^{5}$ Departamento de Solos, Universidade Federal do Paraná, Curitiba, PR, Brasil.

*Autor para correspondência - newton.lucena-costa@embrapa.br
\end{abstract}

\section{Resumo}

Avaliaram-se os efeitos de níveis de correção da fertilidade do solo (testemunha, calagem, adubação e calagem + adubação) e da idade de rebrota $(21,28,35,42,49,56,63,70,77$ e 84 dias) sobre a dinâmica de crescimento e rendimento de forragem de Trachypogon plumosus em Roraima. $\mathrm{O}$ aumento da idade de rebrota resultou em maiores rendimentos de matéria seca (MS), taxa absoluta de crescimento (TAC), taxa de assimilação líquida (TAL), razão de área foliar (RAF) e índice de área foliar (IAF), ocorrendo o inverso quanto à taxa média de crescimento (TMC). A gramínea mostrou-se responsiva à melhoria da fertilidade do solo. A calagem + adubação ou a adubação proporcionaram maiores rendimentos de MS (1.934 e $\left.1.661 \mathrm{~kg} \mathrm{ha}^{-1}\right)$, TAC (36,6 e 31,5 kg ha-1 $\left.\mathrm{dia}^{-1}\right)$, TMC (32,5 e 27,9 $\left.\mathrm{kg} \mathrm{ha}^{-1} \mathrm{dia}^{-1}\right)$, TAL $\left(4,993\right.$ e 4,152 $\left.\mathrm{g} / \mathrm{m}^{2}\right)$, RAF $\left(152,9\right.$ e $\left.140,9 \mathrm{~cm}^{2} / \mathrm{g}\right)$ e IAF $(2,42 \mathrm{e} 2,14)$. Para otimizar a eficiência de utilização da forragem produzida e reduzir perdas por senescência da gramínea, o período mais adequado de sua utilização, durante o período chuvoso, situa-se entre 56 e 63 dias com o uso de adubação e calagem + adubação e entre 63 a 70 dias para a testemunha e a calagem.

Palavras-chave: área foliar; matéria seca; taxa de crescimento.

\begin{abstract}
We assessed the effects of levels of soil fertility correction (control, liming, fertilization and liming + fertilization) and of regrowth ages $(21,28,35,42,49,56,63,70,77$, and 84 days) on growth dynamic and forage yield of Trachypogon plumosus in the Roraima's savannas. Dry matter (DM) yields, absolute growth rate (AGR), net assimilation rate (NAR), and leaf area ratio (LAR) increased consistently with regrowth age, while the crop growth rate (CGR) was inversely proportional to regrowth age. The grass showed high responsivity to soil fertility improvement. Liming + fertilization or fertilization alone provided higher DM yields (1,934 and 1,661 kg ha-1), AGR (36.6 and $31.5 \mathrm{~kg}$ $\mathrm{ha}^{-1}$.day), CGR (32.5 and $27.9 \mathrm{~kg} \mathrm{ha}^{-1}$.day), NAR (4.993 e $\left.4.152 \mathrm{~g} / \mathrm{m}^{2}\right)$, LAR $\left(152.9\right.$ and $\left.140.9 \mathrm{~cm}^{2} / \mathrm{g}\right)$,
\end{abstract}


and LAI (2.42 and 2.14). Regrowth intervals of 56 to 63 days for liming + fertilized and fertilized pastures, and 63 to 70 days for control or liming pastures were appropriate to provide maximum forage yields, growth rates and to prevent larger losses relative to senescence and leaf death.

Keywords: dry matter; growth rate; leaf area.

Enviado em: 28 maio 2012

Aceito em: 29 fevereiro 2016

\section{Introdução}

Nos cerrados de Roraima, as pastagens nativas representam a fonte mais econômica para alimentação dos rebanhos. Apesar de limitações quantitativas e qualitativas, decorrentes da baixa fertilidade natural dos solos, historicamente, as pastagens nativas proporcionaram o suporte alimentar para a exploração pecuária, que passou a constituir, ao longo dos anos, a principal atividade econômica de Roraima ${ }^{(1)}$. O pastejo contínuo com taxa de lotação variável, extensivo e desvinculado do ritmo estacional das pastagens, tem contribuição direta para os baixos índices produtivos dos rebanhos ${ }^{(2)}$. $\mathrm{O}$ excesso de lotação animal e a falta de reposição dos nutrientes extraídos constituem as principais causas do esgotamento da fertilidade do solo do ecossistema e, consequentemente, da redução da capacidade de suporte e do potencial produtivo das pastagens nativas ${ }^{(3)}$.

Nas áreas planas e não inundáveis das pastagens nativas, onde a gramínea Trachypogon plumosus constitui 80 à 90\% de sua composição botânica, a produção animal pode ser muito baixa, sendo necessário de 6 a 10 ha para cada bovino adulto, o que inviabiliza economicamente a atividade pecuária, desde que não sejam implementadas práticas para o seu melhoramento ${ }^{(2)}$.

A gramínea Trachypogon plumosus apresenta ciclo perene, hábito de crescimento cespitoso, plantas com 40 a $60 \mathrm{~cm}$ de altura e folhas pilosas. No entanto, são escassas as informações sobre o seu potencial produtivo e quanto à melhoria das condições do ambiente de produção, notadamente quanto à correção da fertilidade do solo, com a utilização de práticas de manejo mais sustentáveis ${ }^{(4)}$.

A produção primária líquida é a variável de maior interesse quando se avalia o rendimento de ecossistemas de pastagens, sendo os índices de crescimento de grande valia para o esclarecimento dos mecanismos morfofisiológicos responsáveis pela produção de biomassa ${ }^{(5-6)}$. As variações no acúmulo de biomassa, durante a estação de crescimento, permitem o monitoramento da dinâmica da produção fotossintética efetiva, resultado do balanço entre fotossíntese, respiração e perdas por senescência de tecidos e órgãos ${ }^{(4)}$. Neste contexto, deve-se procurar o ponto de equilíbrio entre produtividade e qualidade, visando assegurar os requerimentos nutricionais dos animais e garantindo, simultaneamente, a maximização da eficiência dos processos de utilização e conversão da forragem produzida ${ }^{(3-7)}$.

Neste trabalho foram avaliados os efeitos de níveis de correção da fertilidade do solo e da idade de rebrota sobre a dinâmica da produção de forragem de Trachypogon plumosus nos cerrados de Roraima.

\section{Material e Métodos}

O experimento foi conduzido em uma pastagem nativa de T. plumosus, localizada em Boa Vista, Roraima ( $60^{\circ} 43^{\prime}$ 'de longitude oeste e $2^{\circ} 45^{\prime}$ de latitude norte), a qual não estava submetida a nenhuma prática de manejo. O clima da região, segundo a classificação de Köppen, é Awi, com precipitação anual de $1.600 \mathrm{~mm}$, sendo que $80 \%$ ocorrem nos seis meses do período chuvoso (abril a setembro). O período experimental foi de maio a agosto de 2011, que corresponde à estação chuvosa (Tabela 1). 
Tabela 1:Precipitação, temperaturas mínimas, máximas e médias e radiação solar registradas durante o período experimental. Boa Vista, Roraima, 2011

\begin{tabular}{|c|c|c|c|c|c|}
\hline \multirow[t]{2}{*}{ Meses } & \multirow{2}{*}{$\begin{array}{l}\text { Precipitação } \\
\text { (mm) }\end{array}$} & \multicolumn{3}{|c|}{ Temperatura $\left({ }^{\circ} \mathrm{C}\right)$} & \multirow{2}{*}{$\begin{array}{c}\text { Radiação Solar } \\
\mathbf{M J} / \mathbf{m}^{2}\end{array}$} \\
\hline & & Mínima & Máxima & Média & \\
\hline Maio & 692,9 & 23,0 & 30,8 & 26,9 & 376,9 \\
\hline Junho & 383,8 & 22,7 & 31,7 & 27,2 & 381,8 \\
\hline Julho & 389,2 & 22,2 & 32,1 & 27,1 & 432,4 \\
\hline Agosto & 234,8 & 23,8 & 32,9 & 28,4 & 458,8 \\
\hline
\end{tabular}

O solo da área experimental era um Latossolo Amarelo, textura média, com as seguintes características químicas, na profundidade de $0-20 \mathrm{~cm}: \mathrm{pH}_{\mathrm{H} 2 \mathrm{O}}=5,1 ; \mathrm{P}=1,1 \mathrm{mg} \mathrm{dm}{ }^{-3} ; \mathrm{Ca}+\mathrm{Mg}=0,51 \mathrm{cmol}_{\mathrm{c}} \cdot \mathrm{dm}^{-3}$; $\mathrm{K}=0,03 \mathrm{cmol}_{\mathrm{c}} \cdot \mathrm{dm}^{-3} ; \mathrm{Al}=0,39 \mathrm{cmol}_{\mathrm{c}} \mathrm{dm}^{-3} ; \mathrm{H}+\mathrm{Al}=2,43 \mathrm{cmol}_{c} \cdot \mathrm{dm}^{-3} ;$ matéria orgânica $=10,6 \mathrm{~g} \mathrm{dm}^{-}$ 3; $\mathrm{SB}=0,54 \mathrm{cmol}_{\mathrm{c}} \cdot \mathrm{dm}^{-3} \mathrm{e} \mathrm{V}=18,2 \%$. O delineamento experimental foi em blocos ao acaso com três repetições e os tratamentos arranjados em um fatorial 4 x 10. Foram avaliados quatro níveis de correção da fertilidade do solo (testemunha, calagem, adubação e calagem + adubação) e dez idades de rebrota $(21,28,35,42,49,56,63,70,77$ e 84 dias após a uniformização da pastagem a $5 \mathrm{~cm}$ acima do solo). O tamanho das parcelas foi de 5,0 x 4,0 m, sendo a área útil de $12 \mathrm{~m}^{2}$. A calagem foi realizada 30 dias antes do rebaixamento da pastagem, visando elevar a $40 \%$ a saturação de bases (600 $\mathrm{kg} \mathrm{ha}^{-1}$ de calcário dolomítico - PRNT $=100 \%$ ). A adubação constou de $50 \mathrm{~kg} \mathrm{ha}^{-1}$ de $\mathrm{N}$ (ureia), $50 \mathrm{~kg}$ ha $^{-1}$ de $\mathrm{P}_{2} \mathrm{O}_{5}$ (superfosfato triplo), $50 \mathrm{~kg} \mathrm{ha}^{-1}$ de $\mathrm{K}_{2} \mathrm{O}$ (cloreto de potássio) e $30 \mathrm{~kg} \mathrm{ha}^{-1}$ de $\mathrm{S}$ (enxofre elementar), aplicados a lanço após o rebaixamento da pastagem.

Os rendimentos de massa de matéria seca (MS) foram estimados através de cortes mecânicos, realizados a uma altura de $5 \mathrm{~cm}$ acima do solo. O material colhido foi pesado e colocado para secar em estufa de ventilação forçada de ar a $65^{\circ} \mathrm{C}$ por 72 horas. A taxa absoluta de crescimento (TAC) foi calculada dividindo-se o rendimento de MS pelo respectivo período de rebrota. A taxa média de crescimento (TMC), taxa de crescimento relativo (TCR), taxa de assimilação líquida (TAL) e razão de área foliar (RAF) foram calculadas segundo Pereira et al. ${ }^{(8)}$ :

$\mathrm{TMC}=\mathrm{P}_{2}-\mathrm{P}_{1} / \mathrm{T}_{2}-\mathrm{T}_{1}\left(\mathrm{~kg} \mathrm{ha}^{-1}\right.$.dia de $\left.\mathrm{MS}\right)$

$\mathrm{TCR}=\mathrm{LnP}_{2}-\mathrm{LnP}_{1} / \mathrm{T}_{2}-\mathrm{T}_{1}$ (g/g.dia)

$\mathrm{TAL}=\mathrm{P}_{2}-\mathrm{P}_{1} / \mathrm{T}_{2}-\mathrm{T}_{1} \times \operatorname{Ln} \mathrm{A}_{2}-\mathrm{LnA}_{1} / \mathrm{A}_{2}-\mathrm{A}_{1}\left(\mathrm{~g} / \mathrm{m}^{2}\right.$.dia $)$

$\mathrm{RAF}=\mathrm{A} / \mathrm{P}\left(\mathrm{cm}^{2} \mathrm{~g}^{-1}\right)$

Em que, $\mathrm{P}_{2}$ e $\mathrm{P}_{1} ; \mathrm{A}_{2}$ e $\mathrm{A}_{1}$ e, $\mathrm{T}_{2}$ e $\mathrm{T}_{1}$, representam, respectivamente, a produtividade de $\mathrm{MS}\left(\mathrm{kg} \mathrm{ha}^{-1}\right), \mathrm{o}$ intervalo de tempo (dias) e a área foliar $\left(\mathrm{cm}^{2}\right.$ ou $\left.\mathrm{m}^{2}\right)$ entre duas amostragens.

Para o cálculo da área foliar, em cada idade de rebrota foram coletadas amostras de folhas verdes completamente expandidas, com área aproximada de 200 a $300 \mathrm{~cm}^{2}$, sendo essa estimada com o auxílio de um planímetro ótico eletrônico (Li-Cor, modelo LI-3100C). Após a estimativa da área, as amostras eram levadas à estufa com circulação forçada de ar a $65^{\circ} \mathrm{C}$ até atingirem peso constante, obtendo-se a MS foliar. A área foliar específica (AFE) foi determinada por meio da relação entre a área de folhas verdes e a sua $\mathrm{MS}\left(\mathrm{m}^{2} / \mathrm{g} \mathrm{MS}\right.$ foliar). O índice de área foliar (IAF) foi determinado a partir do produto entre a MS total das folhas verdes (g de $\left.\mathrm{MS} / \mathrm{m}^{2}\right)$ e a AFE $\left(\mathrm{m}^{2} / \mathrm{g}\right.$ de $\mathrm{MS}$ foliar).

Os dados foram submetidos à análise de variância e de regressão, considerando-se o nível de significância de 5\% de probabilidade, utilizando-se o programa Sisvar. Para se estimar a resposta dos parâmetros avaliados às idades de rebrota, em função dos níveis de correção da fertilidade do solo, a escolha dos modelos de regressão baseou-se na significância dos coeficientes linear e quadrático, por meio do teste "t", de Student, ao nível de 5\% de probabilidade. 


\section{Resultados e Discussão}

A gramínea mostrou-se responsiva à melhoria do ambiente de produção, apesar de sua boa adaptação aos solos de baixa fertilidade de cerrados. Os maiores rendimentos de MS foram registrados com a calagem + adubação (1.934 $\left.\mathrm{kg} \mathrm{ha}^{-1}\right)$ e adubação $\left(1.661 \mathrm{~kg} \mathrm{ha}^{-1}\right)$, semelhantes entre si $(\mathrm{P}>0,05)$. $\mathrm{O}$ efeito da calagem foi menos acentuado $\left(1.087 \mathrm{~kg} \mathrm{ha}^{-1}\right)$, porém superior $(\mathrm{P}<0,05)$ à testemunha $(710$ $\mathrm{kg} \mathrm{ha}^{-1}$ ) (Figura 1). Em pastagens de Trachypogon vestitus adubadas com $50 \mathrm{~kg} \mathrm{ha}^{-1}$ de $\mathrm{N}, 50 \mathrm{~kg}$ ha $^{-1}$ de $\mathrm{P}_{2} \mathrm{O}_{5}, 50 \mathrm{~kg} \mathrm{ha}^{-1}$ de $\mathrm{K}_{2} \mathrm{O}, 120 \mathrm{~kg} \mathrm{ha}^{-1}$ de calcário dolomítico e $20 \mathrm{~kg} \mathrm{ha}^{-1}$ de $\mathrm{S}$, Costa et al. ${ }^{(4)}$ estimaram $4.550 \mathrm{~kg} \mathrm{ha}^{-1}$, em comparação a 2.840 e $3.111 \mathrm{~kg} \mathrm{ha}^{-1}$, respectivamente, na ausência de $\mathrm{N}$ ou de adubação. Em pastagens de Axonopus purpusii, a aplicação conjunta de nitrogênio (100 kg de N $\left.\mathrm{ha}^{-1}\right)$, fósforo $\left(36 \mathrm{~kg}\right.$ de $\left.\mathrm{P}_{2} \mathrm{O}_{5} \mathrm{ha}^{-1}\right)$ e potássio $\left(60{\mathrm{~kg} \mathrm{de} \mathrm{K}_{2} \mathrm{O} \mathrm{ha}}^{-1}\right)$ resultou em incrementos superiores a $460 \%$ no seu rendimento de forragem (554 vs. $3.108 \mathrm{~kg}^{2}$ de MS ha $\left.{ }^{-1}\right)^{(9)}$. Utilizando níveis semelhantes de fertilização em pastagens de T. plumosus + T. vestitus, Barger et al. ${ }^{(10)}$ constataram acréscimos de 71; 17 e 126\%, respectivamente, para a aplicação de $\mathrm{N}\left(7.180 \mathrm{~kg}\right.$ de $\left.\mathrm{MS} \mathrm{ha}^{-1}\right), \mathrm{P}+\mathrm{K}(4.900 \mathrm{~kg}$ de MS $\left.\mathrm{ha}^{-1}\right)$ e N + P + K (9.490 kg de MS ha-1), comparando-se ao tratamento controle (4.200 kg de MS ha-1). Para Paspalum regnellii, gramínea nativa da América do Sul, Primavesi et al. ${ }^{(11)}$ constataram maiores rendimentos de MS com a aplicação de $210 \mathrm{~kg}$ de $\mathrm{N} \mathrm{ha}^{-1}, 240 \mathrm{~kg}$ de $\mathrm{P}_{2} \mathrm{O}_{5}$ ha- e $190 \mathrm{~kg}$ de $\mathrm{K}_{2} \mathrm{O}^{-1} \mathrm{ha}^{-1}$. Craine et al..$^{(12)}$ e Ries e Shugart ${ }^{(13)}$ obtiveram acréscimos superiores a 187 e $121 \%$, respectivamente, nos rendimentos de forragem de gramíneas nativas dos cerrados da África do Sul, com a aplicação de $200 \mathrm{~kg}$ de $\mathrm{N} \mathrm{ha}^{-1} \mathrm{ano}^{-1}$ e $100 \mathrm{~kg}$ de $\mathrm{P} \mathrm{ha}^{-1}$ ano $^{-1}$, isoladas ou combinadas. Tendências semelhantes foram reportadas por D'Antonio e Mack $^{(14)}$ para pastagens nativas com predominância de Melinis minutiflora, em que a aplicação conjunta de $100 \mathrm{~kg} \mathrm{de} \mathrm{N} \mathrm{ha}^{-1}$ e $100 \mathrm{~kg}$ de $\mathrm{P} \mathrm{ha}^{-1}$ promoveu incrementos nos rendimentos de MS de 37,9 e 51,1\% (5.345 $\left.\mathrm{kg} \mathrm{ha}^{-1}\right)$, em comparação à aplicação isolada de $\mathrm{N}$ $\left(3.876 \mathrm{~kg} \mathrm{ha}^{-1}\right)$ ou P $\left(2.127 \mathrm{~kg} \mathrm{ha}^{-1}\right)$.

$\mathrm{O}$ efeito da idade de rebrota foi ajustado ao modelo quadrático e os máximos rendimentos registrados aos 78,9 (1.003 kg ha-1); 80,6 (1.340 $\left.\mathrm{kg} \mathrm{ha}^{-1}\right) ; 76,4\left(1.759 \mathrm{~kg} \mathrm{ha}^{-1}\right)$ e 73,4 dias (2.116 $\left.\mathrm{kg} \mathrm{ha}^{-1}\right)$ para testemunha, calagem, adubação e calagem + adubação, respectivamente (Figura 1). Os valores registrados foram superiores aos relatados por Mata et al. ${ }^{(15)}, 1.124$ e $1.417 \mathrm{~kg} \mathrm{ha}^{-1}$, respectivamente, para pastagens de T. plumosus roçadas ou queimadas anualmente e semelhantes aos reportados por Mata et al. ${ }^{(15)}$, na Venezuela, para pastagens de Trachypogon spp., submetidas a diferentes intervalos entre cortes, $\left(1.654 ; 2.309\right.$ e $2.631 \mathrm{~kg} \mathrm{ha}^{-1}$ de MS, respectivamente para cortes a cada 28, 49 e 63 dias). Para T. vestitus, Mata et al. ${ }^{(2)}$ constataram maiores rendimentos de MS com cortes aos $56(3.349 \mathrm{~kg}$ $\left.\mathrm{ha}^{-1}\right)$ ou 126 dias $\left(3.124 \mathrm{~kg} \mathrm{ha}^{-1}\right)$, comparativamente a 224 dias $\left(2.867 \mathrm{~kg} \mathrm{ha}^{-1}\right)$.

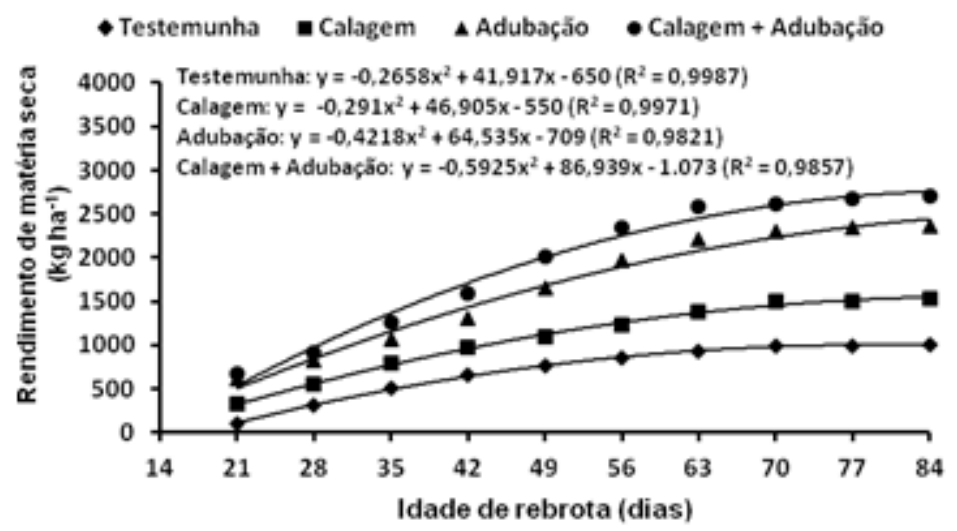

Figura 1: Rendimento de forragem de Trachypogon plumosus, em função dos niveis de correção da fertilidade do solo e das idades de rebrota. 
As maiores TAC e TMC foram registradas com a calagem + adubação (36,6 e 32,5 kg ha-1 $\mathrm{dia}^{-1}$ de MS) e adubação $\left(31,5\right.$ e 27,9 $\mathrm{kg} \mathrm{ha}^{-1}$ dia $^{-1}$ de MS), semelhantes entre si $(\mathrm{P}>0,05)$ e superiores à calagem (20,6 e 20,7 kg ha-1 dia ${ }^{-1}$ de MS) e à testemunha (13,1 e 15,7 $\mathrm{kg} \mathrm{ha}^{-1} \mathrm{dia}^{-1}$ de MS) (Figuras 2 e 3$)$.

O efeito da idade de rebrota sobre a TAC foi ajustado ao modelo quadrático de regressão e os máximos valores obtidos aos 56,5 (14,8 $\mathrm{kg} \mathrm{ha}^{-1} \mathrm{dia}^{-1} \mathrm{de}$ MS); 53,3 (22,9 $\left.\mathrm{kg} \mathrm{ha}^{-1} \mathrm{dia}^{-1} \mathrm{de} \mathrm{MS}\right) ; 53,8$ (33,6 kg $\mathrm{ha}^{-1} \mathrm{dia}^{-1}$ de MS) e 54,2 dias (40,6 $\mathrm{kg} \mathrm{ha}^{-1}$ dia de MS), respectivamente, para testemunha, calagem, adubação e calagem + adubação.

Para a TMC, o ajuste foi exponencial e os maiores valores constatados no período entre 28 (45,7 kg $\mathrm{ha}^{-1} \mathrm{dia}^{-1} \mathrm{de}$ MS) e 42 dias (35,7 $\left.\mathrm{kg} \mathrm{ha}^{-1} \mathrm{dia}^{-1} \mathrm{de} \mathrm{MS}\right)$. Esses valores foram superiores aos estimados por Sarmiento ${ }^{(3)}$ para Axonopus purpusii (11,7 kg ha-1 $\mathrm{dia}^{-1} \mathrm{de}$ MS) e Panicum laxum $\left(7,2 \mathrm{~kg} \mathrm{ha}^{-1} \mathrm{dia}^{-1} \mathrm{de}\right.$ MS). Costa et al. ${ }^{(16)}$ sugeriram como período mais adequado de utilização da pastagem aquele em que as TAC e as TMC são equivalentes, de modo a maximizar o acúmulo líquido de forragem e evitar as perdas por senescência quando as TAC são decrescentes e as variações na TMC são pequenas. Neste contexto, o período entre 49 e 56 dias com o uso de adubação e calagem + adubação e 63 e 70 dias para a testemunha e a calagem seriam recomendados para o pastejo, otimizando a TAC e a TMC da gramínea. Ademais, evidencia-se o efeito positivo da melhoria da fertilidade do solo na antecipação do uso da pastagem.

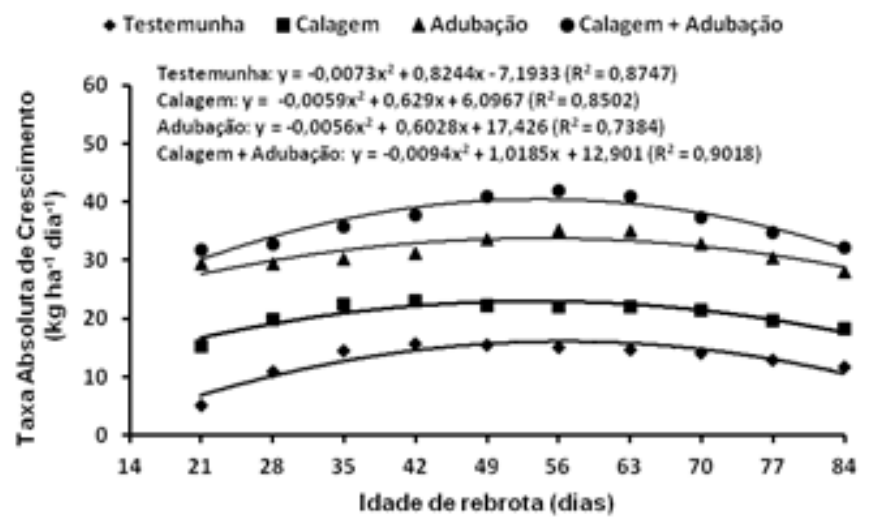

Figura 2: Taxa absoluta de crescimento de Trachypogon plumosus, em função dos niveis de correção da fertilidade do solo e das idades de rebrota.

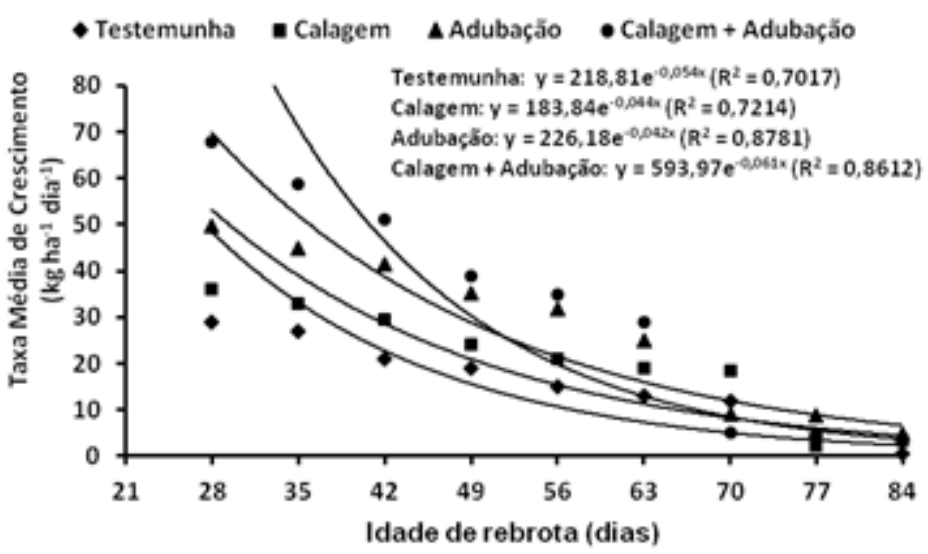

Figura 3: Taxa média de crescimento de Trachypogon plumosus, em função dos niveis de correção da fertilidade do solo e das idades de rebrota. 
A TCR não foi afetada $(\mathrm{P}>0,05)$ pelos níveis de correção da fertilidade do solo, enquanto que a TAL e a RAF apresentaram maiores valores com a calagem + adubação $\left(4,993 \mathrm{~g} / \mathrm{m}^{2}\right.$.dia e $\left.152,9 \mathrm{~cm}^{2} / \mathrm{g}\right)$ e adubação $\left(4,152 \mathrm{~g} / \mathrm{m}^{2}\right.$.dia e 140,9 $\left.\mathrm{cm}^{2} / \mathrm{g}\right)$, semelhantes entre si ( $\left.\mathrm{P}>0,05\right)$ (Figuras 4, 5 e 6$)$. Andrade et al. ${ }^{(5)}$ não constataram efeitos positivos de níveis de $\mathrm{N}$ e $\mathrm{K}$ sobre as TCR, TAL e RAF de Pennisetum purpureum cv. Napier, enquanto que Ries e Shugart ${ }^{(13)}$ constataram acréscimos significativos na taxa de fotossíntese líquida de Panicum maximum com a aplicação isolada de $200 \mathrm{~kg}$ de $\mathrm{N} \mathrm{ha}^{-1}(52,48$ $\mu \mathrm{mol} \mathrm{m} \mathrm{m}^{-2}{\left.\mathrm{se} \mathrm{CO}_{2}\right) \text { e } 100 \mathrm{~kg} \text { de } \mathrm{P} \mathrm{ha}}^{-1}\left(38,45 \mu \mathrm{mol} \mathrm{m}^{-2} \mathrm{~s}^{-1}\right.$ de $\left.\mathrm{CO}_{2}\right)$ ou conjunta $\left(41,30 \mu \mathrm{mol} \mathrm{m}^{-2} \mathrm{~s}^{-1}\right.$

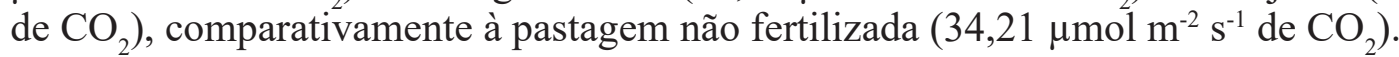

As relações entre idades de rebrota e TCR e TAL foram ajustadas ao modelo exponencial e os maiores valores constatados no período entre $28\left(0,0924 \mathrm{~g} / \mathrm{g}\right.$.dia e $13,411 \mathrm{~g} / \mathrm{m}^{2}$.dia $)$ e 42 dias $(0,0432 \mathrm{~g} / \mathrm{g}$.dia e $6,236 \mathrm{~g} / \mathrm{m}^{2}$.dia). Para pastagens de T. vestitus, Sarmiento ${ }^{(3)}$ reportaram TCR de 0,061; 0,166 e 0,303 g/g.dia, respectivamente, para 30, 132 e 178 dias de rebrota. Para a RAF, a resposta foi quadrática e os máximos valores registrados aos $59,1\left(114,3 \mathrm{~cm}^{2} / \mathrm{g}\right) ; 58,8\left(124,4 \mathrm{~cm}^{2} / \mathrm{g}\right) ; 59,8\left(140,9 \mathrm{~cm}^{2} / \mathrm{g}\right)$ e 59,4 dias $\left(152,9 \mathrm{~cm}^{2} / \mathrm{g}\right)$, respectivamente, para a testemunha, calagem, adubação e calagem + adubação.

A TAL representa a diferença entre a MS produzida pela fotossíntese e a consumida pela respiração. Os decréscimos na TAL e RAF com o aumento da idade de rebrota decorrem da diminuição da capacidade fotossintética líquida do dossel, em função do sombreamento mútuo das folhas e da maior taxa respiratória das plantas, com reflexos diretos sobre a TCR que é o produto entre a TAL e a RAF. A taxa de fotossíntese líquida de folhas de P. maximum cv. Tanzânia aos 33 dias de idade foi reduzida em $25 \%\left(29,0 \mu \mathrm{mol} \mathrm{m}^{-2} \mathrm{~s}^{-1}\right.$ de $\left.\mathrm{CO}_{2}\right)$ comparativamente a folhas aos 11 dias de idade $\left(38,6 \mu \mathrm{mol} \mathrm{m}^{-2} \mathrm{~s}^{-1}\right.$ de $\left.\mathrm{CO}_{2}\right)^{(17)}$. Baruch et al. ${ }^{(18)}$ concluíram que a redução na capacidade fotossintética de gramíneas com rota metabólica $\mathrm{C}_{4}$, em savanas da Venezuela, foi consequência não apenas da diminuição do teor de nitrogênio, mas também do aumento do auto-sombreamento no dossel. Oliveira et al. ${ }^{(19)}$ constataram drásticas reduções nas TAL, RAF e TCR de Cynodon spp., a partir dos 35 dias de rebrota, sugerindo a utilização de suas pastagens em torno desse período de descanso, visando prevenir maiores perdas de forragem por senescência e morte de folhas. Os valores obtidos foram semelhantes aos reportados por Gomide e Gomide ${ }^{(7)}$ e Alexandrino et al. ${ }^{(20)}$ para P. maximum cvs. Vencedor, Tanzânia e Mombaça e superiores aos estimados por Baruch e Bilbao ${ }^{(21)}$ para T. plumosus $\left(0,0511 \mathrm{~g} / \mathrm{g}\right.$.dia; $7,815 \mathrm{~g} / \mathrm{m}^{2}$.dia e $\left.92,5 \mathrm{~cm}^{2} / \mathrm{g}\right)$ e Silva et al. ${ }^{(22)}$ para Brachiaria brizantha cv. MG-5 $\left(0,0859 \mathrm{~g} / \mathrm{g}\right.$.dia; $9,877 \mathrm{~g} / \mathrm{m}^{2}$.dia e 72,9 $\mathrm{cm}^{2} / \mathrm{g}$ ). Pereira et al. ${ }^{(23)}$ sugeriram valores de TCR entre 0,02 e $0,5 \mathrm{~g} / \mathrm{g}$.dia como ideais para a maioria de espécies $\mathrm{C}_{4}$, sob diversas condições ambientais e de manejo.

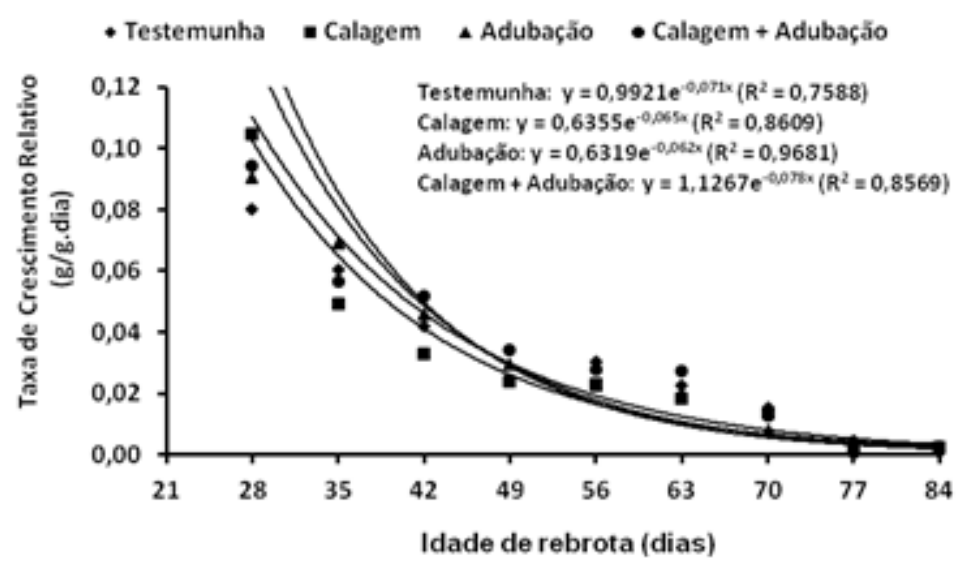

Figura 4: Taxa de crescimento relativo de Trachypogon plumosus, em função dos niveis de correção da fertilidade do solo e das idades de rebrota. 


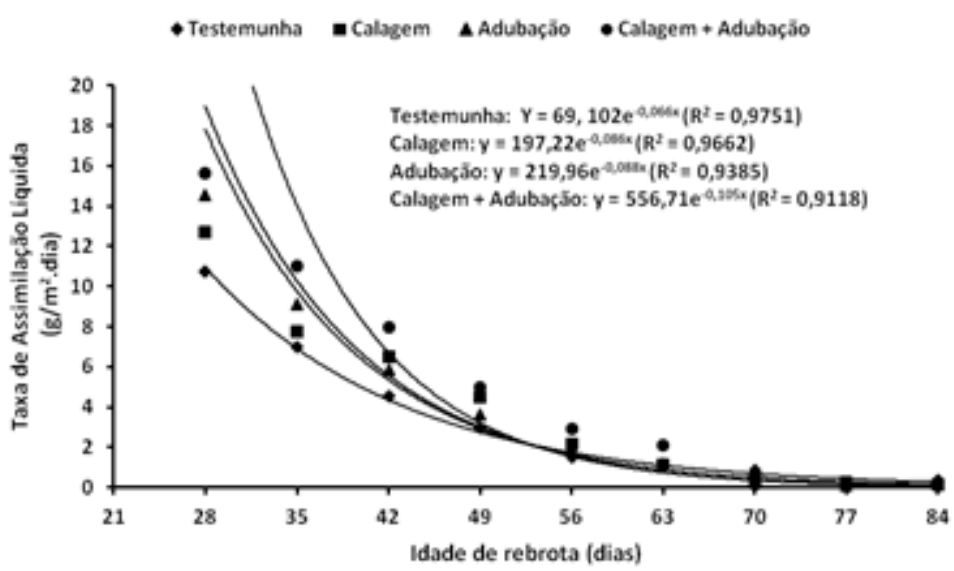

Figura 5: Taxa de assimilação liquida de Trachypogon plumosus, em função dos níveis de correção da fertilidade do solo e das idades de rebrota.

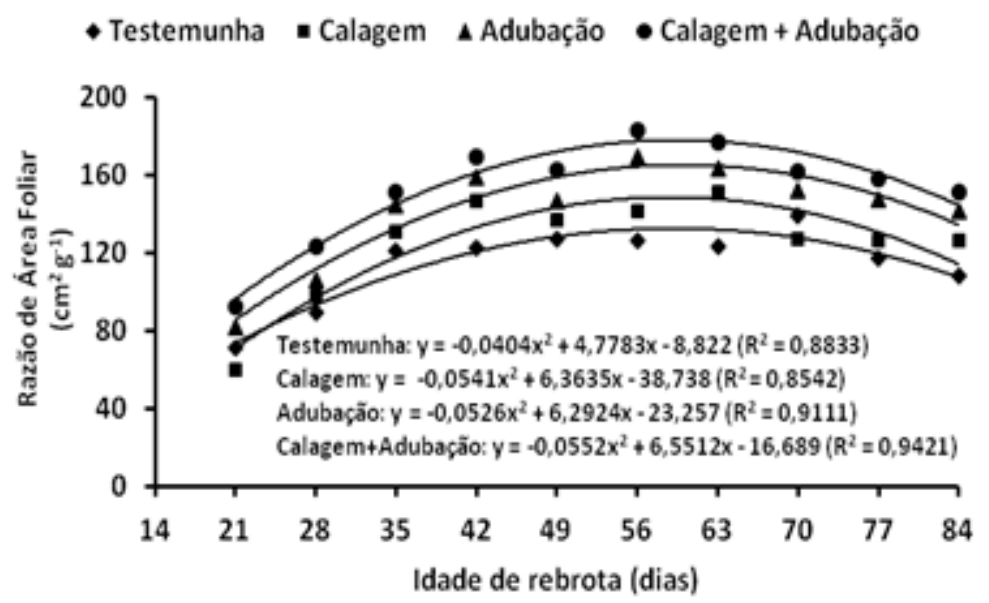

Figura 6: Razão de área foliar de Trachypogon plumosus, em função dos níveis de correção da fertilidade do solo e das idades de rebrota.

O IAF foi positivamente influenciado $(\mathrm{P}<0,05)$ pela calagem + adubação $(2,42)$ e adubação $(2,14)$, enquanto que a calagem $(1,59)$ e a testemunha $(1,08)$ proporcionaram os menores valores (Figura 7). Como o IAF representa a síntese das características morfogênicas e estruturais da gramínea, ele reflete o balanço dos processos que determinam a oferta (fotossíntese) e a demanda (respiração, acúmulo de reservas, síntese e senescência de tecidos) de fotoassimilados, que estabelecem o ritmo de crescimento da pastagem ${ }^{(6,24)}$. A ação positiva da melhoria da fertilidade do solo sobre a taxa de alongamento foliar, comprimento final de folha e densidade populacional de perfilhos expressa-se diretamente sobre o IAF da pastagem ${ }^{(24,25)}$. O efeito da idade de rebrota sobre o IAF foi quadrático com os máximos valores registrados aos 81,5 (1,53), 77,3 (2,25), 75,0 (2,83) e 73,8 dias $(2,59)$, respectivamente, para testemunha, calagem, adubação e calagem + adubação. Os valores de IAF obtidos foram superiores aos relatados por Baruch e Bilbao ${ }^{(21)}$ para T. plumosus $(1,31)$ e por Sarmiento et al. ${ }^{(9)}$ para $A$. purpusii $(1,54)$, Hymenachne amplexicaulis $(1,02)$ e $P$. laxum $(0,72)$, gramíneas nativas dos cerrados da Venezuela. Para gramíneas de hábito de crescimento cespitoso, o IAF crítico, em 
que $95 \%$ da luz incidente seriam interceptadas, normalmente varia entre 3,0 e 5,0 $0^{(3,25)}$, sendo esta condição atendida a partir dos 77 e 63 dias de rebrota, respectivamente, com a aplicação da adubação e calagem + adubação. Gramíneas com maior habilidade competitiva são aquelas que desenvolvem uma arquitetura mais eficiente na interceptação de luz, através da rápida expansão de área foliar e colonização da camada superior do dossel, em decorrência do alongamento da bainha e entrenós do colmo $^{(6-24)}$.

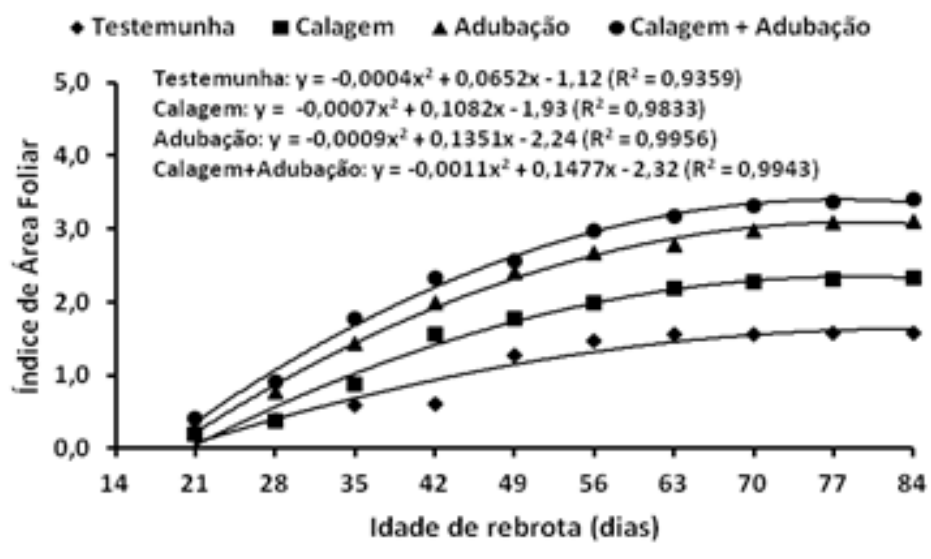

Figura 7: Índice de área foliar de Trachypogon plumosus, em função dos níveis de correção da fertilidade do solo e das idades de rebrota.

\section{Conclusões}

A gramínea apresenta respostas positivas à melhoria das condições do ambiente de produção. Os níveis de correção da fertilidade do solo e as idades de rebrota afetam o rendimento, o padrão de acúmulo de forragem e as taxas de crescimento da gramínea. O uso da adubação ou da calagem + adubação antecipa o período de utilização da pastagem.

Visando otimizar a eficiência de utilização da forragem produzida e reduzir as perdas por senescência foliar da gramínea, o período mais adequado de utilização de suas pastagens, durante o período chuvoso, situa-se entre 56 e 63 dias com o uso de adubação e calagem + adubação e entre 63 e 70 dias quando se usa apenas a calagem ou nenhuma correção.

\section{Referências}

1. Costa NL, Ganluppi, V, Moraes A, Bendahan AB. Produtividade de forragem e características morfogênicas e estruturais de Axonopus aureus nos cerrados de Roraima. Amazônia Ciência \& Desenvolvimento, 2011; 6 (12):7-22.

2. Mata D, Herrera P, Birbe B. Sistemas de producción animal con bajos insumos para las sabanas de Trachypogon sp. Ecotropicos, 1996; 9 (2):83-100.

3. Sarmiento G. Adaptative strategies of perennial grasses in South American savannas. Journal of Vegetation Science, 1992; 3:325-336.

4. Costa NL, Gianluppi V, Moraes A. Morfogênese de Trachypogon vestitus submetido à queima, nos cerrados de Roraima. Ciência Animal Brasileira, 2012; 13 (1): 41-48. 
5. Andrade AC, Fonseca DM, Lopes RS, Nascimento Júnior D, Cecon PR, Queiroz DS, Pereira DH, Reis ST. Análise de crescimento do capim-elefante 'Napier' adubado e irrigado. Ciência e Agrotecnologia, 2005; 29 (2): 415-423.

6. Santos MER, Fonseca DM, Gomes, VM. Correlações entre características morfogênicas e estruturais em pastos de capim-braquiária. Ciência Animal Brasileira, 2012; 13 (1):49-56.

7. Gomide CAM, Gomide JA. Morfogênese de cultivares de Panicum maximum Jacq. Revista Brasileira de Zootecnia, 2000; 29 (2):341-348.

8. Pereira OG, Oliveira MA, Pinto JC, Santos MER, Ribeiro KG, Cecon PR. Análise de crescimento do capim Coastcross-1 sob adubação nitrogenada em duas idades de rebrotação. Revista Brasileira de Zootecnia, 2011; 40 (10): 2121-2128

9. Sarmiento G, Silva MP, Naranjo ME, Pinillos M. Nitrogen and phosphorus as limiting factors for growth and primary production in a flooded savanna in the Venezuelan Llanos. Journal of Tropical Ecology, 2006; 22 (3):203-212.

10. Barger NN, D'Antonio CM, Ghneim T, Brink K, Cuevas EN. Nutrient limitation to primary productivity in a secondary savanna in Venezuela. Biotropica, 2002; 34 (4):493-501.

11. Primavesi O, Primavesi AC, Batista LA, Godoy R. Adubação e produção de Paspalum em dois níveis de fertilidade de Latossolo Vermelho-Amarelo: estabelecimento e manutenção. Ciência e Agrotecnologia, 2008; 32 (1):242-250.

12. Craine JM, Morrow C, Stock WD. Nutrient concentration ratios and co-limitation in South African grasslands. New Phytologist, 2008; 179:829-836.

13. Ries LP, Shugart HH. Nutrient limitations on understory grass productivity and carbon assimilation in a African woodland savanna. Journal of Arid Environment, 2008; 72:1423-1430.

14. D'Antonio CM, Mack MC. Nutrient limitation in a fire-derived, nitrogen-rich Hawaiian grassland. Biotropica, 2006; 38 (4):458-467.

15. Mata D, Moreno E, Rojs NR. Efecto de la edad sobre la composición química del Trachypogon spp. en una sabana del Sureste del Estado Guarico. Zootecnia Tropical, 1985; 3 (1):29-48.

16. Costa NL, Moraes A, Gianluppi V, Bendahan, AB. Avaliação da rebrota natural de pastagens de Trachypogon plumosus nos cerrados de Roraima. Scientia Agraria Paranaensis, 2014; 13 (1):57-64.

17. Braga GJ, Mello ACL, Pedreira CGS, Medeiros HR. Fotossíntese e taxa diária de produção de forragem em pastagens de capim-tanzânia sob lotação intermitente. Pesquisa Agropecuária Brasileira, 2009; 44 (1):84-91.

18. Baruch Z, Nassar JM, Bubis J. Quantitative trait, genetic, environmental, and geographical distances among populations of Trachypogon plumosus in Neotropical savanna. Diversity and Distributions, 2004; 10 (1):283-292.

19. Oliveira MA, Pereira OG, Huaman CAM, Garcia R, Gomide JA, Cecón, P, Silveira PR Características morfogênicas e estruturais do capim-bermuda 'Tifton 85' (Cynodon spp.) em diferentes idades de rebrota. Revista Brasileira de Zootecnia, 2000; 29 (6):1939-1948.

20. Alexandrino E, Gomide JA, Gomide CAM. Crescimento e desenvolvimento do dossel de Panicum maximum cv. Mombaça. Revista Brasileira de Zootecnia, 2005; 36 (4):2164-2173.

21. Baruch Z, Bilbao B. Effects of fire and defoliation on the life history of native and invader $\mathrm{C}_{4}$ grass in a Neotropical savanna. Oecologia, 1999; 119 (3):510-520.

22. Silva AC, Ferreira LA, Silva AA, Ferreira FA. Análise de crescimento de Brachiaria brizantha submetida a doses reduzidas de fluazipop-p-butil. Planta Daninha, 2005; 23 (1):85-91. 
23. Pereira OG, Rovetta R, Ribeiro KG, Santos MER, Fonseca DM, Cecon, PR. Crescimento do capim-tifton 85 sob doses de nitrogênio e alturas de corte. Revista Brasileira de Zootecnia, 2012; 41 (1):30-35.

24. Nabinger C, Carvalho P.C.F. Ecofisiología de sistemas pastoriles: aplicaciones para su sustentabilidad. Agrociencia, 2009; 3 (1):18-27.

25. Costa NL, Moraes A, Carvalho PCF, Monteiro ALG, Oliveira, RA. Produtividade de forragem e morfogênese de Trachypogon plumosus sob diferentes níveis de desfolhação. Revista Científica de Produção Animal, 2012; 14 (2):169-172. 